\title{
Applications of Normal S-Iterative Method to a Nonlinear Integral Equation
}

\author{
Faik Gürsoy \\ Department of Mathematics, Faculty of Science and Letters, Yildiz Technical University, Davutpasa Campus, Esenler, \\ 34220 Istanbul, Turkey
}

Correspondence should be addressed to Faik Gürsoy; faikgursoy02@hotmail.com

Received 4 June 2014; Accepted 22 July 2014; Published 10 August 2014

Academic Editor: M. Mursaleen

Copyright (c) 2014 Faik Gürsoy. This is an open access article distributed under the Creative Commons Attribution License, which permits unrestricted use, distribution, and reproduction in any medium, provided the original work is properly cited.

It has been shown that a normal S-iterative method converges to the solution of a mixed type Volterra-Fredholm functional nonlinear integral equation. Furthermore, a data dependence result for the solution of this integral equation has been proven.

\section{Introduction}

The scientists working in almost every field of science are faced with nonlinear problems, because nature itself is intrinsically nonlinear. Such problems can be modelled as nonlinear mathematical equations. Solving nonlinear equations is, of course, considered to be a matter of the uttermost importance in mathematics and its manifold applications. There are numerous systematic approaches which are classified as direct and iterative methods to solve such equations in the existing literature. Indeed, by using direct methods, finding solutions to a complicated nonlinear equation can be an almost insurmountable challenge. In this context, iterative methods have become very important mathematical tools for finding solutions to a nonlinear equation. For a comprehensive review and references to the extensive literature on the iterative methods, the interested reader may refer to some recent works [1-8].

Recently, Sahu [9] and Khan [10], who was probably unaware of Sahu's work, introduced the following iterative process which has been called normal S-iterative method and Picard-Mann hybrid iterative process by Sahu and Khan, respectively, and hereinafter referred to as the "normal Siterative method."

Definition 1. Let $X$ be an ambient space and let $T$ be a selfmap of $X$. A normal S-iterative method is defined by

$x_{0} \in X$,

$$
\begin{aligned}
& x_{n+1}=T y_{n}, \\
& y_{n}=\left(1-\xi_{n}\right) x_{n}+\xi_{n} T x_{n}, \quad n \in \mathbb{N},
\end{aligned}
$$

where $\left\{\xi_{n}\right\}_{n=0}^{\infty}$ is a real sequence in $[0,1]$ satisfying certain control condition(s).

It has been shown both analytically and numerically in $[9,10]$ that iterative method (1) converges at a rate faster than all Picard [11], Mann [12], and Ishikawa [13] iterative processes in the sense of Berinde [14] for the class of contraction mappings.

This iterative method, due to its simplicity and fastness, has attracted the attention of many researchers and has been examined in various aspects; see [15-20].

In this paper, inspired by the performance and achievements of normal S-iterative method (1), we will give some of its applications. We will show that normal S-iterative method (1) converges strongly to the solution of the following mixed type Volterra-Fredholm functional nonlinear integral equation which was considered in [21]:

$$
\begin{gathered}
x(t)=F\left(t, x(t), \int_{a_{1}}^{t_{1}} \cdots \int_{a_{m}}^{t_{m}} K(t, s, x(s)) d s,\right. \\
\left.\int_{a_{1}}^{b_{1}} \cdots \int_{a_{m}}^{b_{m}} H(t, s, x(s)) d s\right),
\end{gathered}
$$


where $\left[a_{1} ; b_{1}\right] \times \cdots \times\left[a_{m} ; b_{m}\right]$ is an interval in $\mathbb{R}^{m}, K, H$ : $\left[a_{1} ; b_{1}\right] \times \cdots \times\left[a_{m} ; b_{m}\right] \times\left[a_{1} ; b_{1}\right] \times \cdots \times\left[a_{m} ; b_{m}\right] \times \mathbb{R} \rightarrow \mathbb{R}$ continuous functions, and $F:\left[a_{1} ; b_{1}\right] \times \cdots \times\left[a_{m} ; b_{m}\right] \times \mathbb{R}^{3} \rightarrow \mathbb{R}$.

Also we give a data dependence result for the solution of integral equation (2) with the help of normal S-iterative method (1).

We end this section with some known results which will be useful in proving our main results.

Theorem 2 (see [21]). We suppose that the following conditions are satisfied:

$\left(A_{1}\right) K, H \in C\left(\left[a_{1} ; b_{1}\right] \times \cdots \times\left[a_{m} ; b_{m}\right] \times\left[a_{1} ; b_{1}\right] \times \cdots \times\right.$ $\left.\left[a_{m} ; b_{m}\right] \times \mathbb{R}\right)$;

$\left(A_{2}\right) F \in C\left(\left[a_{1} ; b_{1}\right] \times \cdots \times\left[a_{m} ; b_{m}\right] \times \mathbb{R}^{3}\right) ;$

$\left(A_{3}\right)$ there exist nonnegative constants $\alpha, \beta$, and $\gamma$ such that

$$
\begin{aligned}
& \left|F\left(t, u_{1}, v_{1}, w_{1}\right)-F\left(t, u_{2}, v_{2}, w_{2}\right)\right| \\
& \quad \leq \alpha\left|u_{1}-u_{2}\right|+\beta\left|v_{1}-v_{2}\right|+\gamma\left|w_{1}-w_{2}\right|
\end{aligned}
$$

for all $t \in\left[a_{1} ; b_{1}\right] \times \cdots \times\left[a_{m} ; b_{m}\right], u_{i}, v_{i}, w_{i} \in \mathbb{R}, i=1,2$;

$\left(A_{4}\right)$ there exist nonnegative constants $L_{K}$ and $L_{H}$ such that

$$
\begin{aligned}
& |K(t, s, u)-K(t, s, v)| \leq L_{K}|u-v|, \\
& |H(t, s, u)-H(t, s, v)| \leq L_{H}|u-v|,
\end{aligned}
$$

for all $t, s \in\left[a_{1} ; b_{1}\right] \times \cdots \times\left[a_{m} ; b_{m}\right], u, v \in \mathbb{R}$;

$$
\left(A_{5}\right) \alpha+\left(\beta L_{K}+\gamma L_{H}\right)\left(b_{1}-a_{1}\right) \cdots\left(b_{m}-a_{m}\right)<1 .
$$

Then (2) has a unique solution $x^{*} \in C\left(\left[a_{1} ; b_{1}\right] \times \cdots \times\right.$ $\left.\left[a_{m} ; b_{m}\right]\right)$.

Lemma 3 (see [22]). Let $\left\{\beta_{n}\right\}_{n=0}^{\infty}$ be a nonnegative sequence for which one assumes there exists $n_{0} \in \mathbb{N}$, such that for all $n \geq n_{0}$ one has satisfied the inequality

$$
\beta_{n+1} \leq\left(1-\mu_{n}\right) \beta_{n}+\mu_{n} \gamma_{n}
$$

where $\mu_{n} \in(0,1)$, for all $n \in \mathbb{N}, \sum_{n=0}^{\infty} \mu_{n}=\infty$, and $\gamma_{n} \geq 0$, for all $n \in \mathbb{N}$. Then the following inequality holds:

$$
0 \leq \lim \sup _{n \rightarrow \infty} \beta_{n} \leq \lim \sup _{n \rightarrow \infty} \gamma_{n} .
$$

\section{Main Results}

Theorem 4. One opines that all conditions $\left(A_{1}\right)-\left(A_{5}\right)$ in Theorem 2 are performed. Let $\left\{\xi_{n}\right\}_{n=0}^{\infty} \subset[0,1]$ be a real sequence satisfying $\sum_{n=0}^{\infty} \xi_{n}=\infty$. Then (2) has a unique solution, say $x^{*}$, in $C\left(\left[a_{1} ; b_{1}\right] \times \cdots \times\left[a_{m} ; b_{m}\right]\right)$ and normal $S$ iterative method (1) converges to $x^{*}$.
Proof. We consider the Banach space $B=C\left(\left[a_{1} ; b_{1}\right] \times \cdots \times\right.$ $\left.\left[a_{m} ; b_{m}\right],\|\cdot\|_{C}\right)$, where $\|\cdot\|_{C}$ is Chebyshev's norm. Let $\left\{x_{n}\right\}_{n=0}^{\infty}$ be an iterative sequence generated by normal $S$-iterative method (1) for the operator $A: B \rightarrow B$ defined by

$$
\begin{gathered}
A(x)(t)=F\left(t, x(t), \int_{a_{1}}^{t_{1}} \cdots \int_{a_{m}}^{t_{m}} K(t, s, x(s)) d s,\right. \\
\left.\int_{a_{1}}^{b_{1}} \cdots \int_{a_{m}}^{b_{m}} H(t, s, x(s)) d s\right) .
\end{gathered}
$$

We will show that $x_{n} \rightarrow x^{*}$ as $n \rightarrow \infty$.

From (1), (2), and assumptions $\left(A_{1}\right)-\left(A_{4}\right)$, we have that

$$
\begin{aligned}
& \left\|x_{n+1}-x^{*}\right\| \\
& =\left\|A y_{n}-x^{*}\right\|=\left|A\left(y_{n}\right)(t)-A\left(x^{*}\right)(t)\right| \\
& =\mid F\left(t, y_{n}(t), \int_{a_{1}}^{t_{1}} \ldots \int_{a_{m}}^{t_{m}} K\left(t, s, y_{n}(s)\right) d s,\right. \\
& \left.\int_{a_{1}}^{b_{1}} \ldots \int_{a_{m}}^{b_{m}} H\left(t, s, y_{n}(s)\right) d s\right) \\
& -F\left(t, x^{*}(t), \int_{a_{1}}^{t_{1}} \ldots \int_{a_{m}}^{t_{m}} K\left(t, s, x^{*}(s)\right) d s,\right.
\end{aligned}
$$

$$
\left.\int_{a_{1}}^{b_{1}} \cdots \int_{a_{m}}^{b_{m}} H\left(t, s, x^{*}(s)\right) d s\right)
$$

$$
\begin{aligned}
& \leq \alpha\left|y_{n}(t)-x^{*}(t)\right| \\
& +\beta \mid \int_{a_{1}}^{t_{1}} \cdots \int_{a_{m}}^{t_{m}} K\left(t, s, y_{n}(s)\right) d s \\
& \quad-\int_{a_{1}}^{t_{1}} \ldots \int_{a_{m}}^{t_{m}} K\left(t, s, x^{*}(s)\right) d s \mid \\
& +\gamma \mid \int_{a_{1}}^{b_{1}} \ldots \int_{a_{m}}^{b_{m}} H\left(t, s, y_{n}(s)\right) d s \\
& \quad-\int_{a_{1}}^{b_{1}} \ldots \int_{a_{m}}^{b_{m}} H\left(t, s, x^{*}(s)\right) d s \mid \\
& \leq \alpha\left|y_{n}(t)-x^{*}(t)\right| \\
& +\beta \int_{a_{1}}^{t_{1}} \ldots \int_{a_{m}}^{t_{m}}\left|K\left(t, s, y_{n}(s)\right)-K\left(t, s, x^{*}(s)\right)\right| d s \\
& +\gamma \int_{a_{1}}^{b_{1}} \ldots \int_{a_{m}}^{b_{m}}\left|H\left(t, s, y_{n}(s)\right)-H\left(t, s, x^{*}(s)\right)\right| d s
\end{aligned}
$$




$$
\begin{aligned}
& \leq \alpha\left|y_{n}(t)-x^{*}(t)\right|+\beta \int_{a_{1}}^{t_{1}} \cdots \int_{a_{m}}^{t_{m}} L_{K}\left|y_{n}(s)-x^{*}(s)\right| d s \\
& \quad+\gamma \int_{a_{1}}^{b_{1}} \cdots \int_{a_{m}}^{b_{m}} L_{H}\left|y_{n}(s)-x^{*}(s)\right| d s \\
& \leq\left[\alpha+\left(\beta L_{K}+\gamma L_{H}\right) \prod_{i=1}^{m}\left(b_{i}-a_{i}\right)\right]\left\|y_{n}-x^{*}\right\|,
\end{aligned}
$$$$
\left\|y_{n}-x^{*}\right\|
$$$$
\leq\left(1-\xi_{n}\right)\left|x_{n}(t)-x^{*}(t)\right|
$$$$
+\xi_{n}\left|A\left(x_{n}\right)(t)-A\left(x^{*}\right)(t)\right|
$$$$
=\left(1-\xi_{n}\right)\left|x_{n}(t)-x^{*}(t)\right|
$$$$
+\xi_{n} \mid F\left(t, x_{n}(t), \int_{a_{1}}^{t_{1}} \cdots \int_{a_{m}}^{t_{m}} K\left(t, s, x_{n}(s)\right) d s,\right.
$$$$
\left.\int_{a_{1}}^{b_{1}} \cdots \int_{a_{m}}^{b_{m}} H\left(t, s, x_{n}(s)\right) d s\right)
$$$$
-F\left(t, x^{*}(t), \int_{a_{1}}^{t_{1}} \cdots \int_{a_{m}}^{t_{m}} K\left(t, s, x^{*}(s)\right) d s,\right.
$$$$
\left.\int_{a_{1}}^{b_{1}} \cdots \int_{a_{m}}^{b_{m}} H\left(t, s, x^{*}(s)\right) d s\right)
$$$$
\leq\left(1-\xi_{n}\right)\left|x_{n}(t)-x^{*}(t)\right|+\xi_{n} \alpha\left|x_{n}(t)-x^{*}(t)\right|
$$$$
+\xi_{n} \beta \int_{a_{1}}^{t_{1}} \cdots \int_{a_{m}}^{t_{m}} L_{K}\left|x_{n}(s)-x^{*}(s)\right| d s
$$$$
+\xi_{n} \gamma \int_{a_{1}}^{b_{1}} \cdots \int_{a_{m}}^{b_{m}} L_{H}\left|x_{n}(s)-x^{*}(s)\right| d s
$$$$
\leq\left\{1-\xi_{n}\left(1-\left[\alpha+\left(\beta L_{K}+\gamma L_{H}\right) \prod_{i=1}^{m}\left(b_{i}-a_{i}\right)\right]\right)\right\}
$$$$
\times\left\|x_{n}-x^{*}\right\| .
$$

Combining (8) with (9), we obtain

$$
\begin{aligned}
& \left\|x_{n+1}-x^{*}\right\| \\
& \leq\left[\alpha+\left(\beta L_{K}+\gamma L_{H}\right) \prod_{i=1}^{m}\left(b_{i}-a_{i}\right)\right] \\
& \quad \times\left\{1-\xi_{n}\left(1-\left[\alpha+\left(\beta L_{K}+\gamma L_{H}\right) \prod_{i=1}^{m}\left(b_{i}-a_{i}\right)\right]\right)\right\} \\
& \quad \times\left\|x_{n}-x^{*}\right\|,
\end{aligned}
$$

or, from assumption $\left(A_{5}\right)$,

$$
\begin{aligned}
& \left\|x_{n+1}-x^{*}\right\| \\
& \leq\left\{1-\xi_{n}\left(1-\left[\alpha+\left(\beta L_{K}+\gamma L_{H}\right) \prod_{i=1}^{m}\left(b_{i}-a_{i}\right)\right]\right)\right\} \\
& \quad \times\left\|x_{n}-x^{*}\right\| .
\end{aligned}
$$

Thus, by induction, we get

$$
\begin{aligned}
\left\|x_{n+1}-x\right\| \leq & \left\|x_{0}-x^{*}\right\| \\
& \times \prod_{k=0}^{n}\left\{1-\xi_{k}\left(1-\left[\alpha+\left(\beta L_{K}+\gamma L_{H}\right)\right.\right.\right. \\
& \left.\left.\left.\times \prod_{i=1}^{m}\left(b_{i}-a_{i}\right)\right]\right)\right\} .
\end{aligned}
$$

Since $\xi_{k} \in[0,1]$ for all $k \in \mathbb{N}$, assumption $\left(A_{5}\right)$ yields

$$
1-\xi_{k}\left(1-\left[\alpha+\left(\beta L_{K}+\gamma L_{H}\right) \prod_{i=1}^{m}\left(b_{i}-a_{i}\right)\right]\right)<1 .
$$

Having regard to the fact that $e^{x} \geq 1-x$ for all $x \in[0,1]$, we can rewrite (12) as

$$
\left\|x_{n+1}-x^{*}\right\| \leq\left\|x_{0}-x^{*}\right\| e^{-\left(1-\left[\alpha+\left(\beta L_{K}+\gamma L_{H}\right) \prod_{i=1}^{m}\left(b_{i}-a_{i}\right)\right]\right) \sum_{k=0}^{n} \xi_{k}}
$$

which yields $\lim _{n \rightarrow \infty}\left\|x_{n}-x^{*}\right\|=0$.

We now prove the data dependence of the solution for integral equation (2) with the help of the normal S-iterative method (1).

Let $B$ be as in the proof of Theorem 4 and $T, \widetilde{T}: B \rightarrow B$ two operators defined by

$$
\begin{gathered}
T(x)(t)=F\left(t, x(t), \int_{a_{1}}^{t_{1}} \cdots \int_{a_{m}}^{t_{m}} K(t, s, x(s)) d s,\right. \\
\left.\int_{a_{1}}^{b_{1}} \cdots \int_{a_{m}}^{b_{m}} H(t, s, x(s)) d s\right), \\
\widetilde{T}(x)(t)=F\left(t, x(t), \int_{a_{1}}^{t_{1}} \cdots \int_{a_{m}}^{t_{m}} \widetilde{K}(t, s, x(s)) d s,\right. \\
\left.\int_{a_{1}}^{b_{1}} \ldots \int_{a_{m}}^{b_{m}} \widetilde{H}(t, s, x(s)) d s\right),
\end{gathered}
$$

where $K, \widetilde{K}, H, \widetilde{H} \in C\left(\left[a_{1} ; b_{1}\right] \times \cdots \times\left[a_{m} ; b_{m}\right] \times\left[a_{1} ; b_{1}\right] \times \cdots \times\right.$ $\left.\left[a_{m} ; b_{m}\right] \times \mathbb{R}\right)$.

Theorem 5. Let F, K, and $H$ be defined as in Theorem 2 and let $\left\{x_{n}\right\}_{n=0}^{\infty}$ be an iterative sequence defined by normal 
S-iterative method (1) associated with T. Let $\left\{\tilde{x}_{n}\right\}_{n=0}^{\infty}$ be an iterative sequence generated by

$$
\begin{aligned}
& \tilde{x}_{0} \in B, \\
& \tilde{x}_{n+1}=\widetilde{T} \tilde{y}_{n}, \\
& \tilde{y}_{n}=\left(1-\xi_{n}\right) \tilde{x}_{n}+\xi_{n} \widetilde{T} \widetilde{x}_{n}, \quad n \in \mathbb{N},
\end{aligned}
$$

where $B$ is defined as in the proof of Theorem 4 and $\left\{\xi_{n}\right\}_{n=0}^{\infty}$ is a real sequence in $[0,1]$ satisfying (i) $1 / 2 \leq \xi_{n}$, for all $n \in \mathbb{N}$, and (ii) $\sum_{n=0}^{\infty} \xi_{n}=\infty$. One supposes further that (iii) there exist nonnegative constants $\varepsilon_{1}$ and $\varepsilon_{2}$ such that $\mid K(t, s, u)-$ $\widetilde{K}(t, s, u) \mid \leq \varepsilon_{1}$ and $|H(t, s, u)-\widetilde{H}(t, s, u)| \leq \varepsilon_{2}$, for all $u \in \mathbb{R}$ and for all $t, s \in\left[a_{1} ; b_{1}\right] \times \cdots \times\left[a_{m} ; b_{m}\right]$.

If $x^{*}$ and $\tilde{x}^{*}$ are solutions of corresponding equations (15) and (16), respectively, then one has that

$$
\left\|x^{*}-\tilde{x}^{*}\right\| \leq \frac{3\left(\beta \varepsilon_{1}+\gamma \varepsilon_{2}\right) \prod_{i=1}^{m}\left(b_{i}-a_{i}\right)}{1-\left[\alpha+\left(\beta L_{K}+\gamma L_{H}\right) \prod_{i=1}^{m}\left(b_{i}-a_{i}\right)\right]} .
$$

Proof. Using (1), (15), (16), (17), and assumptions $\left(A_{1}\right)-\left(A_{4}\right)$ and (iii), we obtain

$$
\begin{aligned}
& \left\|x_{n+1}-\tilde{x}_{n+1}\right\| \\
& =\left\|T y_{n}-\tilde{T} \tilde{y}_{n}\right\| \\
& =\mid F\left(t, y_{n}(t), \int_{a_{1}}^{t_{1}} \cdots \int_{a_{m}}^{t_{m}} K\left(t, s, y_{n}(s)\right) d s,\right. \\
& \left.\int_{a_{1}}^{b_{1}} \cdots \int_{a_{m}}^{b_{m}} H\left(t, s, y_{n}(s)\right) d s\right) \\
& -F\left(t, \tilde{y}_{n}(t), \int_{a_{1}}^{t_{1}} \cdots \int_{a_{m}}^{t_{m}} \widetilde{K}\left(t, s, \widetilde{y}_{n}(s)\right) d s,\right. \\
& \left.\int_{a_{1}}^{b_{1}} \cdots \int_{a_{m}}^{b_{m}} \widetilde{H}\left(t, s, \widetilde{y}_{n}(s)\right) d s\right) \\
& \leq \alpha\left|y_{n}(t)-\tilde{y}_{n}(t)\right| \\
& +\beta \int_{a_{1}}^{t_{1}} \cdots \int_{a_{m}}^{t_{m}}\left|K\left(t, s, y_{n}(s)\right)-\widetilde{K}\left(t, s, \widetilde{y}_{n}(s)\right)\right| d s \\
& +\gamma \int_{a_{1}}^{b_{1}} \cdots \int_{a_{m}}^{b_{m}}\left|H\left(t, s, y_{n}(s)\right)-\widetilde{H}\left(t, s, \tilde{y}_{n}(s)\right)\right| d s \\
& \leq \alpha\left|y_{n}(t)-\tilde{y}_{n}(t)\right| \\
& +\beta \int_{a_{1}}^{t_{1}} \cdots \int_{a_{m}}^{t_{m}}\left(\left|K\left(t, s, y_{n}(s)\right)-K\left(t, s, \tilde{y}_{n}(s)\right)\right|\right. \\
& \left.+\left|K\left(t, s, \widetilde{y}_{n}(s)\right)-\widetilde{K}\left(t, s, \widetilde{y}_{n}(s)\right)\right|\right) d s \\
& +\gamma \int_{a_{1}}^{b_{1}} \cdots \int_{a_{m}}^{b_{m}}\left(\left|H\left(t, s, y_{n}(s)\right)-H\left(t, s, \widetilde{y}_{n}(s)\right)\right|\right. \\
& \left.+\left|H\left(t, s, \widetilde{y}_{n}(s)\right)-\widetilde{H}\left(t, s, \widetilde{y}_{n}(s)\right)\right|\right) d s
\end{aligned}
$$

$$
\begin{aligned}
\leq & \alpha\left|y_{n}(t)-\tilde{y}_{n}(t)\right| \\
& +\beta \int_{a_{1}}^{t_{1}} \ldots \int_{a_{m}}^{t_{m}}\left(L_{K}\left|y_{n}(s)-\tilde{y}_{n}(s)\right|+\varepsilon_{1}\right) d s \\
& +\gamma \int_{a_{1}}^{b_{1}} \ldots \int_{a_{m}}^{b_{m}}\left(L_{H}\left|y_{n}(s)-\tilde{y}_{n}(s)\right|+\varepsilon_{2}\right) d s \\
\leq & \alpha\left\|y_{n}-\tilde{y}_{n}\right\|+\beta\left(L_{K}\left\|y_{n}-\tilde{y}_{n}\right\|+\varepsilon_{1}\right) \prod_{i=1}^{m}\left(b_{i}-a_{i}\right) \\
& +\gamma\left(L_{H}\left\|y_{n}-\tilde{y}_{n}\right\|+\varepsilon_{2}\right) \prod_{i=1}^{m}\left(b_{i}-a_{i}\right) \\
\leq & {\left[\alpha+\left(\beta L_{K}+\gamma L_{H}\right) \prod_{i=1}^{m}\left(b_{i}-a_{i}\right)\right]\left\|y_{n}-\tilde{y}_{n}\right\| } \\
& +\left(\beta \varepsilon_{1}+\gamma \varepsilon_{2}\right) \prod_{i=1}^{m}\left(b_{i}-a_{i}\right),
\end{aligned}
$$

$$
\begin{aligned}
& \left\|y_{n}-\tilde{y}_{n}\right\| \\
& \leq\left(1-\xi_{n}\right)\left|x_{n}(t)-\tilde{x}_{n}(t)\right| \\
& +\xi_{n}\left|T\left(x_{n}\right)(t)-\widetilde{T}\left(\tilde{x}_{n}\right)(t)\right| \\
& \leq\left(1-\xi_{n}\right)\left|x_{n}(t)-\tilde{x}_{n}(t)\right| \\
& +\xi_{n}\left\{\alpha\left|x_{n}(t)-\tilde{x}_{n}(t)\right|\right. \\
& +\beta \int_{a_{1}}^{t_{1}} \cdots \int_{a_{m}}^{t_{m}}\left\{L_{K}\left|x_{n}(s)-\tilde{x}_{n}(s)\right|+\varepsilon_{1}\right\} d s \\
& \left.+\gamma \int_{a_{1}}^{b_{1}} \ldots \int_{a_{m}}^{b_{m}}\left\{L_{H}\left|x_{n}(s)-\tilde{x}_{n}(s)\right|+\varepsilon_{2}\right\} d s\right\} \\
& \leq\left\{1-\xi_{n}\left(1-\left[\alpha+\left(\beta L_{K}+\gamma L_{H}\right) \prod_{i=1}^{m}\left(b_{i}-a_{i}\right)\right]\right)\right\} \\
& \times\left\|x_{n}-\tilde{x}_{n}\right\|+\xi_{n}\left(\beta \varepsilon_{1}+\gamma \varepsilon_{2}\right) \prod_{i=1}^{m}\left(b_{i}-a_{i}\right) .
\end{aligned}
$$

Combining (19) with (20) and using assumptions $\left(A_{5}\right)$ and $1 / 2 \leq \xi_{n}$ in the resulting inequality, we get

$$
\begin{aligned}
& \left\|x_{n+1}-\tilde{x}_{n+1}\right\| \\
& \leq\left\{1-\xi_{n}\left(1-\left[\alpha+\left(\beta L_{K}+\gamma L_{H}\right) \prod_{i=1}^{m}\left(b_{i}-a_{i}\right)\right]\right)\right\} \\
& \quad \times\left\|x_{n}-\tilde{x}_{n}\right\|
\end{aligned}
$$




$$
\begin{aligned}
& +\xi_{n}\left(1-\left[\alpha+\left(\beta L_{K}+\gamma L_{H}\right) \prod_{i=1}^{m}\left(b_{i}-a_{i}\right)\right]\right) \\
& \times \frac{3\left(\beta \varepsilon_{1}+\gamma \varepsilon_{2}\right) \prod_{i=1}^{m}\left(b_{i}-a_{i}\right)}{1-\left[\alpha+\left(\beta L_{K}+\gamma L_{H}\right) \prod_{i=1}^{m}\left(b_{i}-a_{i}\right)\right]} .
\end{aligned}
$$

Denote that

$$
\begin{aligned}
& \beta_{n}=\left\|x_{n}-\tilde{x}_{n}\right\|, \\
& \mu_{n}=\xi_{n}\left(1-\left[\alpha+\left(\beta L_{K}+\gamma L_{H}\right) \prod_{i=1}^{m}\left(b_{i}-a_{i}\right)\right]\right) \in(0,1), \\
& \gamma_{n}=\frac{3\left(\beta \varepsilon_{1}+\gamma \varepsilon_{2}\right) \prod_{i=1}^{m}\left(b_{i}-a_{i}\right)}{1-\left[\alpha+\left(\beta L_{K}+\gamma L_{H}\right) \prod_{i=1}^{m}\left(b_{i}-a_{i}\right)\right]} \geq 0 .
\end{aligned}
$$

It is clear that inequality (21) satisfies all conditions in Lemma 3, and hence it follows that

$$
\left\|x^{*}-\tilde{x}^{*}\right\| \leq \frac{3\left(\beta \varepsilon_{1}+\gamma \varepsilon_{2}\right) \prod_{i=1}^{m}\left(b_{i}-a_{i}\right)}{1-\left[\alpha+\left(\beta L_{K}+\gamma L_{H}\right) \prod_{i=1}^{m}\left(b_{i}-a_{i}\right)\right]} .
$$

\section{Conflict of Interests}

The author declares that there is no conflict of interests regarding the publication of this paper.

\section{Acknowledgment}

The author would like to thank the anonymous reviewers for their valuable comments and suggestions to improve the quality of the paper.

\section{References}

[1] C. Chidume, Geometric Properties of Banach Spaces and Nonlinear Iterations, vol. 1965, Springer, London, UK, 2009.

[2] F. Gürsoy and V. Karakaya, "Some convergence and stability results for two new Kirk type hybrid fixed point iterative algorithms," Journal of Function Spaces, vol. 2014, Article ID 684191, 8 pages, 2014.

[3] F. Gürsoy, V. Karakaya, and B. E. Rhoades, "Data dependence results of new multi-step and S-iterative schemes for contractive-like operators," Fixed Point Theory and Applications, vol. 2013, artcile 76, 12 pages, 2013.

[4] H. Kiziltunc and S. Temir, "Convergence theorems by a new iteration process for a finite family of nonself asymptotically nonexpansive mappings with errors in Banach spaces," Computers \& Mathematics with Applications, vol. 61, no. 9, pp. 24802489, 2011.

[5] M. Basarir and A. Sahin, "On the strong and $\Delta$-convergence of new multi-step and S-iteration processes in a CAT (0) space," Journal of Inequalities and Applications, vol. 2013, article 482, 2013.

[6] M. O. Olatinwo, "Convergence and stability results for some iterative schemes," Acta Universitatis Apulensis, no. 26, pp. 225236, 2011.
[7] S. Almezel, Q. H. Ansari, and M. A. Khamsi, Eds., Topics in Fixed Point Theory, Springer, 2014.

[8] V. Berinde, Iterative Approximation of Fixed Points, Springer, Berlin, Germany, 2007.

[9] D. R. Sahu, "Applications of the S-iteration process to constrained minimization problems and split feasibility problems," Fixed Point Theory, vol. 12, no. 1, pp. 187-204, 2011.

[10] S. H. Khan, "A Picard-Mann hybrid iterative process," Fixed Point Theory and Applications, vol. 2013, article 69, 2013.

[11] E. Picard, "Mémoire sur la théorie des équations aux dérivées partielles et la méthode des approximations successives," Journal de Matématiques Pures et Appliquées, vol. 6, pp. 145-210, 1890.

[12] W. R. Mann, "Mean value methods in iteration," Proceedings of the American Mathematical Society, vol. 4, pp. 506-510, 1953.

[13] S. Ishikawa, "Fixed points by a new iteration method," Proceedings of the American Mathematical Society, vol. 44, pp. 147-150, 1974.

[14] V. Berinde, "Picard iteration converges faster than Mann iteration for a class of quasi-contractive operators," Fixed Point Theory and Applications, vol. 2004, no. 2, pp. 97-105, 2004.

[15] D. R. Sahu and A. Petruşel, "Strong convergence of iterative methods by strictly pseudocontractive mappings in Banach spaces," Nonlinear Analysis: Theory, Methods \& Applications, vol. 74, no. 17, pp. 6012-6023, 2011.

[16] N. Hussain, V. Kumar, and M. A. Kutbi, "On rate of convergence of Jungck-type iterative schemes," Abstract and Applied Analysis, vol. 2013, Article ID 132626, 15 pages, 2013.

[17] S. H. Khan, "Fixed points of contractive-like operators by a faster iterative process," WASET International Journal of Mathematical, Computational Science and Engineering, vol. 7, pp. 57-59, 2013.

[18] S. M. Kang, A. Rafiq, and S. Lee, "Convergence analysis of an iterative scheme for Lipschitzian hemicontractive mappings in Hilbert spaces," Journal of Inequalities and Applications, vol. 2013, article 312, 5 pages, 2013.

[19] S. M. Kang, A. Rafiq, and S. Lee, "Strong convergence of an implicit S -iterative process for Lipschitzian hemicontractive mappings," Abstract and Applied Analysis, vol. 2012, Article ID 804745, 7 pages, 2012.

[20] V. Kumar, A. Latif, A. Rafiq, and N. Hussain, "S-iteration process for quasi-contractive mappings," Journal of Inequalities and Applications, vol. 2013, article 206, 15 pages, 2013.

[21] C. Crăciun and M. Şerban, "A nonlinear integral equation via Picard operators," Fixed Point Theory, vol. 12, no. 1, pp. 57-70, 2011.

[22] Ş. M. Şoltuz and T. Grosan, "Data dependence for Ishikawa iteration when dealing with contractive-like operators," Fixed Point Theory and Applications, vol. 2008, Article ID 242916, 7 pages, 2008. 


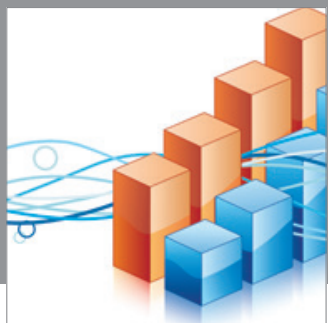

Advances in

Operations Research

mansans

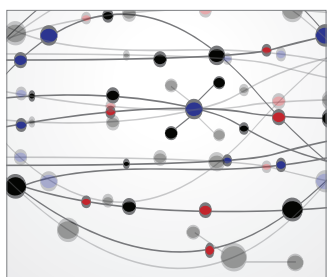

The Scientific World Journal
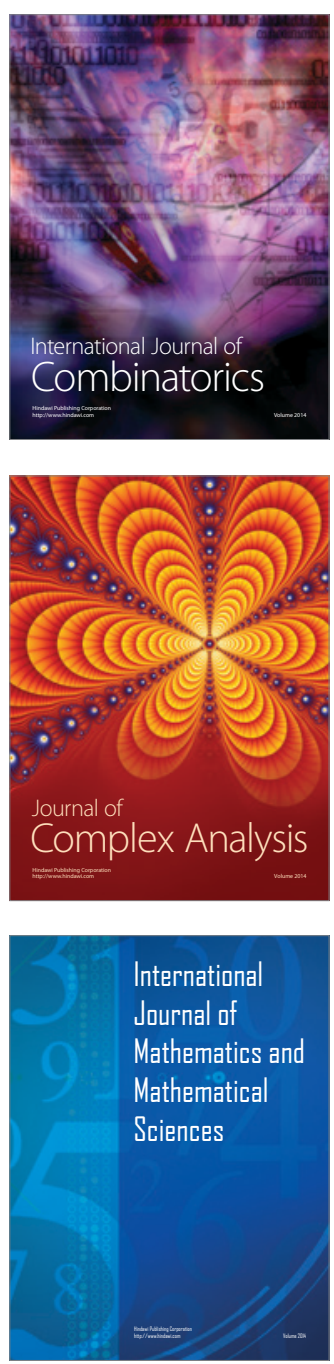
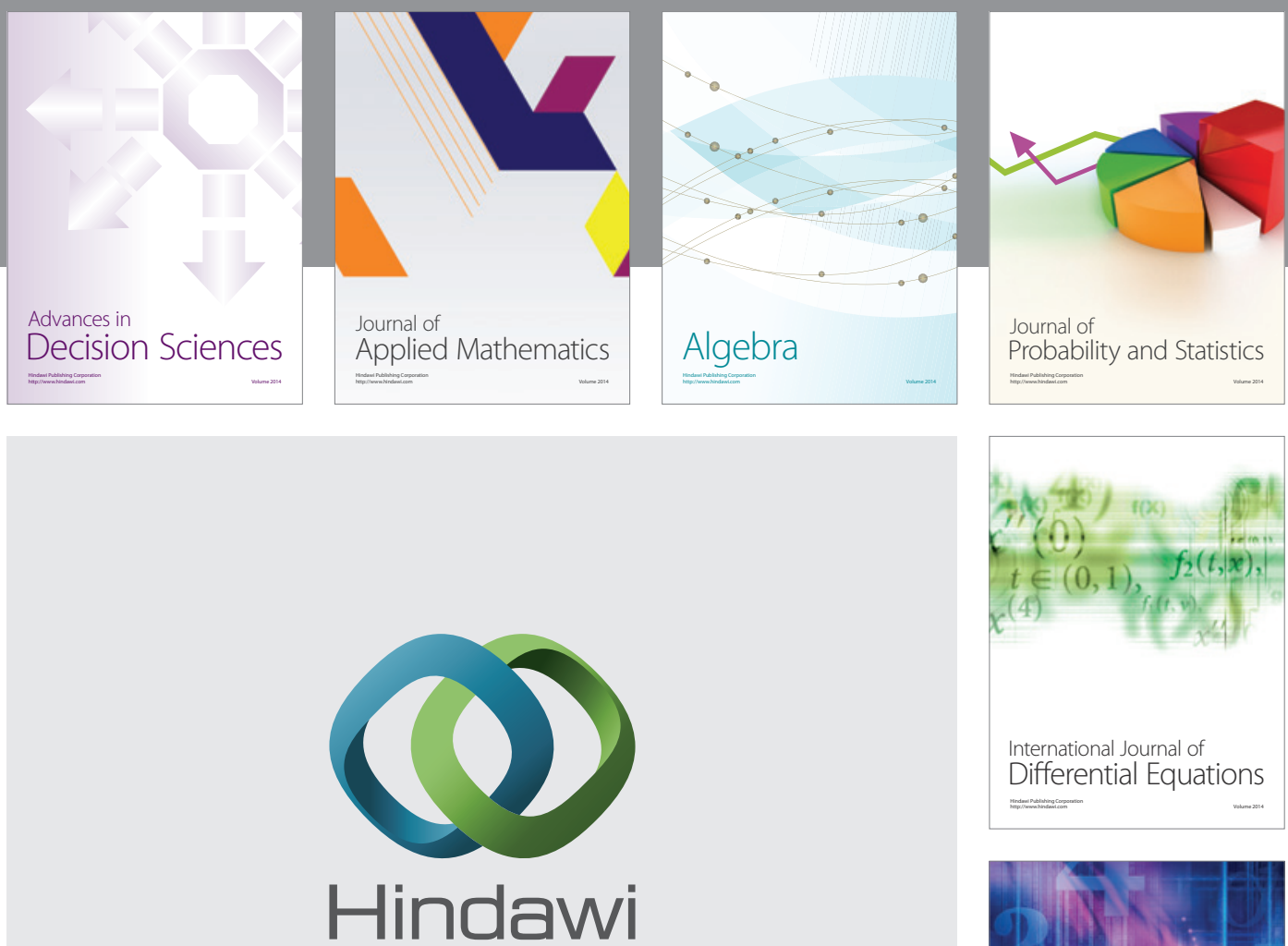

Submit your manuscripts at http://www.hindawi.com
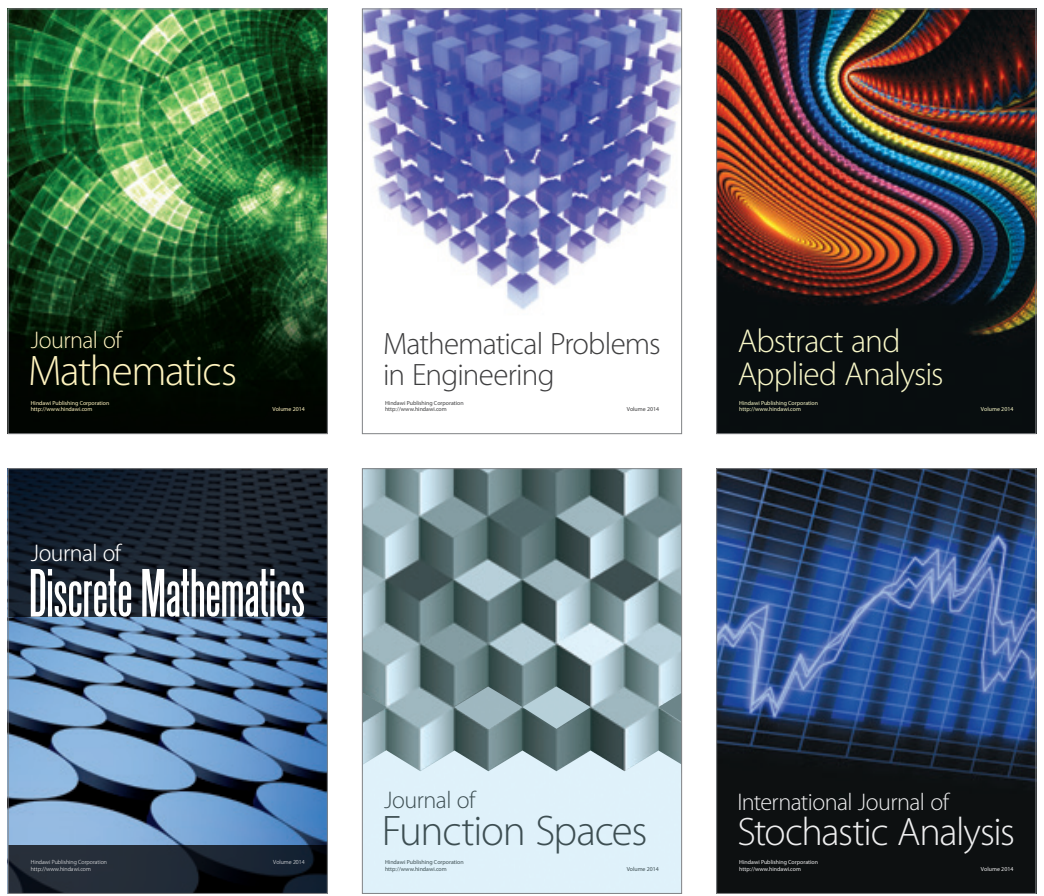

Journal of

Function Spaces

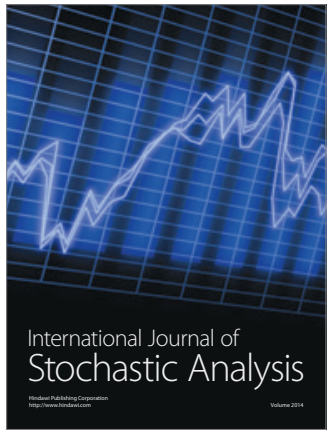

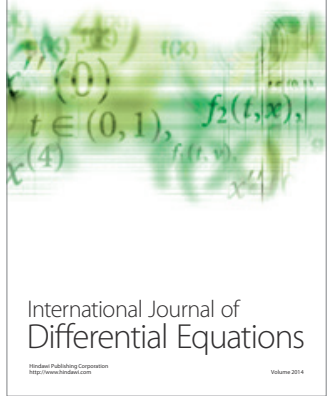
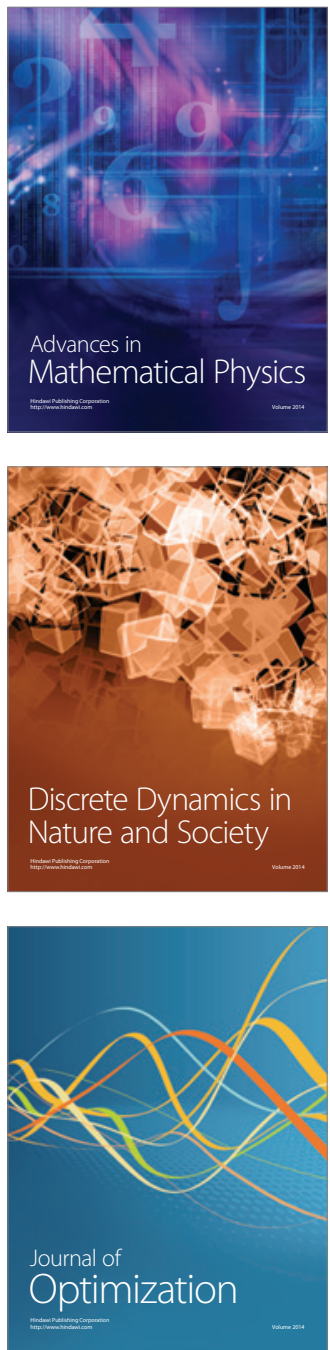